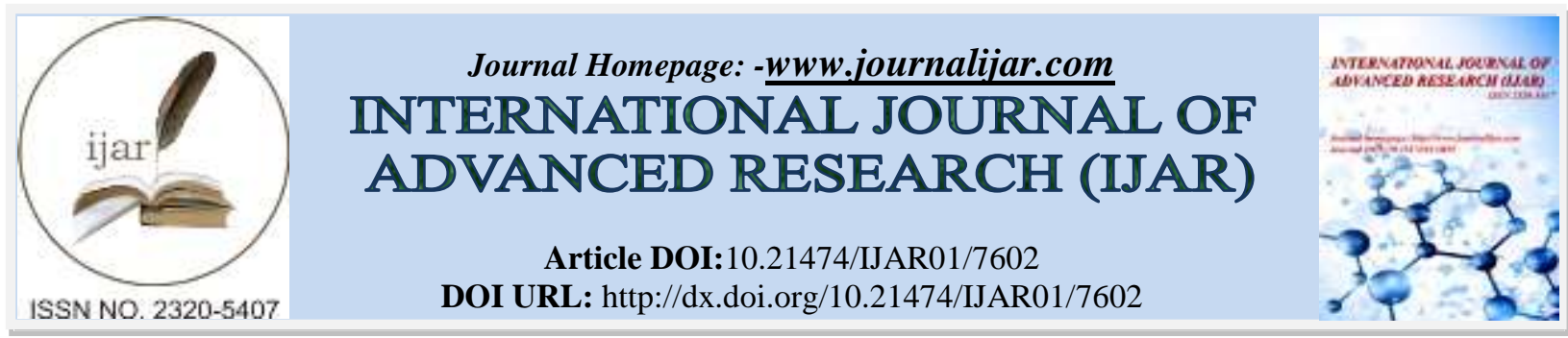

RESEARCH ARTICLE

\title{
IMMEDIATE IMPLANT PLACEMENT IN FRESHLY EXTRACTED SOCKET: A CASE REPORT.
}

\section{Fauzia Tarannum ${ }^{1}$, Kaushik Kumar pandey ${ }^{1}$,Abhishek Gaur ${ }^{2}$, Taruna Patel ${ }^{3}$, Saurabh Patel $^{2}$ and Vivek Gautam ${ }^{3}$.}

1. 1,2Assistant professor, Department of Prosthodontics, Career Post Graduate Institute of Dental Sciences and Hospital, Lucknow, India.

2. Associate professor, Department of Prosthodontics, Career Post Graduate Institute of Dental Sciences and Hospital, Lucknow, India.

3. Private Practitioner

\section{Manuscript Info}

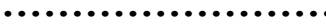

Manuscript History

Received: 20 June 2018

Final Accepted: 22 July 2018

Published: August 2018

Keywords:-

Atraumatic Extraction, Fresh Extraction

Sockets, Immediate implant.

\begin{abstract}
Immediate dental implant placement refers to insertion of an implant directly after a tooth is extracted, whereas de layed positioning occurs at some later time. Implant-supported fixed prostheses are usually attempted before other options such as conventional bridges or removable dentures to avoid damage to adjacent teeth. Stable and predictable esthetic and functional results can be achieved with different surgical and prosthetic management techniques of the hard and soft tissue around implant restorations. It has been suggested that immediate implant placement after tooth extraction preserves the dimensions of the alveolar ridge regardless of the mode of tooth failure. After tooth extraction, the residual alveolar ridge generally provides limited bone volume because of ongoing resorbtion. In this case we extracted mandibular anterior teeth immediately placed the implants.
\end{abstract}

Copy Right, IJAR, 2018,. All rights reserved.

\section{Introduction:-}

Nowadays advances in clinical techniques and biomaterials have facilitated a great expansion in the indications for dental implant treatment options.

Placement of implants immediately following extraction has now become an increasingly common strategy to preserve bone and reduce treatment time. Also, improves esthetics because the soft tissue envelope is preserved ${ }^{1}$.

Since the evolution of the implant, it has been opted as the most preferable and reliable method for functional and esthetic rehabilitation. In the earlier days, the dental implants were used to be placed in the healed alveolar ridges, which used to take minimum 5-6 months after the extraction of teeth. In the year 1989, 1st ever reported immediate dental implant placement was carried out by Lazzara, since then, many of the studies have confirmed the reliability of immediate implants placement. Immediate implant placement, defined as, "the placement of dental implant immediately into fresh extraction socket site after tooth extraction, has been considered a predictable and acceptable procedure". 2 
The replacement of anterior tooth with an implant in freshly extracted socket is a complex surgical procedure mainly due to the cascade of events that follow every tooth extraction.

\section{Indications and Contraindications for Immediate implant Placement:-}

There are numerous indications for tooth replacement with an immediate implant when an adequate amount of bone and soft tissue are available to support it: deciduous tooth, en do don tic failure, caries, deep probing depths due to periodontitis, vertical root fracture, and idiopathic root resorption. Contra indictions to inserting immediate implants include inadequate height or width of bone, lack of soft tissue, ad verse location of nerves, proximity of adjacent teeth, failure to achieve primary stability, and inability to attain a restoratively reasonable position, an gulation or

sink depth of the implant.

\section{Immediate Implant Survival Rates:-}

Implants immediately placed into fresh extraction sockets and healed ridges have similar survival rates (97.3\% to $99 \%)^{4}$.

\section{Considerations For Immediate Implant Placement:-}

Atraumatic Tooth Removal Prior to Implant Insertion:-

Teeth need to be removed atraumatically to preserve the maximum amount of bone before immediate implant placement

Width and Length of an Implant to Attain Primary Stability:-

Depending on the size of the extracted tooth and the implant to be placed, somewhere along the root surface, the implant will usually exceed the diameter of the root and provide mechanical retention of the implant mainly from the base of the socket.

This retention and/or extension of the osteotomy and placement of the implant beyond the apex of the extracted tooth provide primary implant stability. It is advisable to place an implant a minimum of 3 to $5 \mathrm{~mm}$ into bone to attain primary stability if mechanical retention cannot be achieved laterally. ${ }^{5}$

\section{Apicocoronal and Horizontal Placement of Immediate Dental Implants:-}

In general, immediate implants should be placed one mm subcrestally as viewed from the midpoint of the labial plate. As a general rule, platforms of immediate implants should be placed 2 to $3 \mathrm{~mm}$ below the gingival margin to account for vertical bone height resorption (the implant often will be deeper interproximally) ${ }^{6,7}$. If the buccal or lingual plates of bone are thin, the implants should be placed more subcrestally, since there may be an increased amount of bone resorption. Horizontally, implants should not touch the buccal plate of bone because there is a horizontal zone of influence, and if an implant encroaches upon the buccal plate of bone, it will induce resorption.

\section{Clinical Report:-}

A 27 year old male patient reported to the Department of Prosthodontics, Babu Banarsi Das college of Dental Sciences, Lucknow with chief complaint of fractured and grossly carious tooth in lower front region [Fig. 1]. Radiographic examination revealed root stumps with horizontal root fracture and periapical pathology in 31,32 with unfavourable prognosis, 41 and 42 were restored with post and core [Fig. 2]. All the available treatment options were told to the patient and the definitive planned procedure included immediate implant placement in 32 and 41 region to restore 32,31 and 41 teeth. The patient was very concerned about the esthetics and was very interested for the restoration of his teeth and so he opted for the proposed procedure.
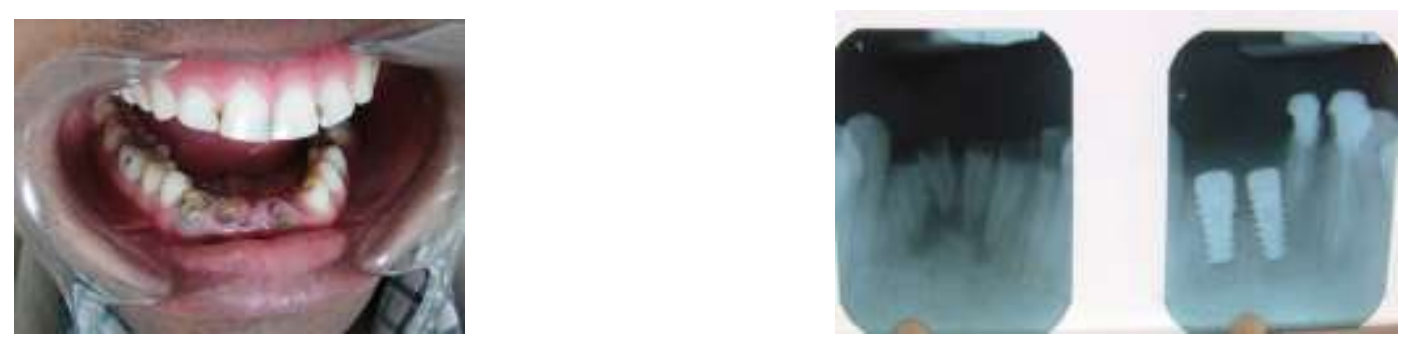


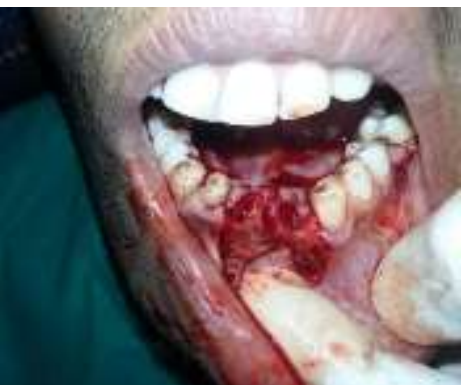

Fig3

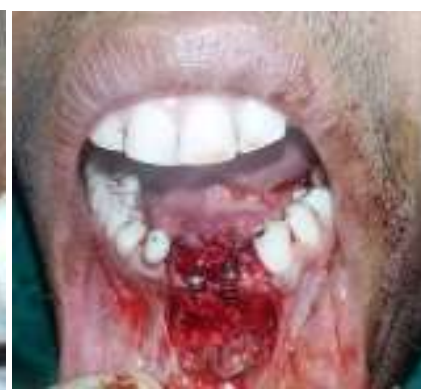

Fig-4

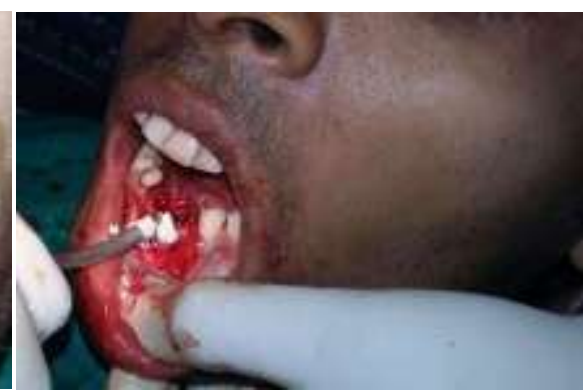

Fig-5

Pre-surgical radiographic evaluation was carried out for appropriate treatment planning. After proper treatment planning endo-osseous implant (Adin Touareg Internal hex square thread, Israel) measuring $4.2 \times 11.5 \mathrm{~mm}$ in dimension was selected. The fractured tooth was atraumatically removed with the help of periotomes. After extraction, the site was thoroughly degranulated using curettes. The socket irrigation was done with Povidine Iodine and carefully examine the intact socket walls. The socket was evaluated for osseous defects.

A sequential drilling was carried out with $2.2,2.8,3.2,3.65 \mathrm{~mm}$. Implant placement was done in the extraction socket with the insertion torque of $45 \mathrm{Ncm}$ [Fig. 3,4]. The first thread of implant was placed $1.5 \mathrm{~mm}$ apical to crestal bone of the socket to gain primary stability ${ }^{5}$. Fenestrated buccal plate were filled with Bio- Oss, Platelet rich fibrin (PRF) were made from patient blood. PRF along with grafts and PTFE barrier membrane (Cytoplast ${ }^{\circ}$ TXT-200) were placed to secure the implant for better osseointegration.(Fig 5,6,7). There is evidence that resorbable barriers without grafting reduces alveolar ridge resorption after tooth extraction ${ }^{8}$. Post-operative periapical radiograph was taken [Fig. 2].Sutures were placed.(Fig 8)

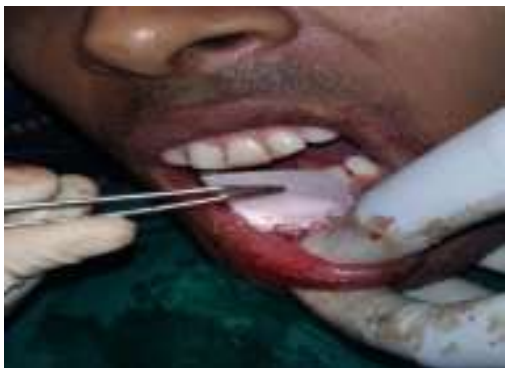

Fig-6

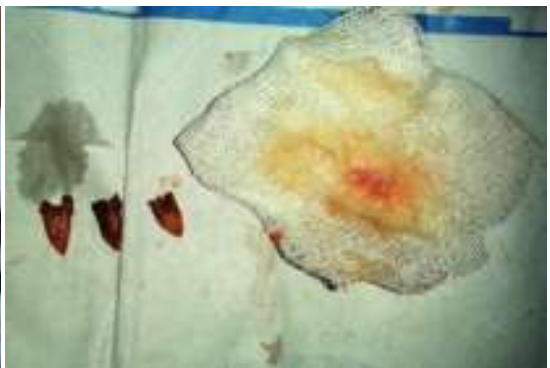

Fig-7

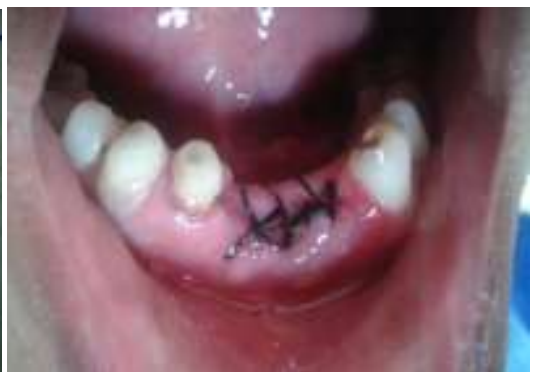

Fig-8

The patient was administered an analgesic (Ibuprofen $800 \mathrm{mg}$, every 4 to 6 hours) for 7 days and an antibiotic (Augmentin $625 \mathrm{mg}, 2$ times daily) for 7 days. After 3 months gingival former was placed and left for 2 weeks ${ }^{9}$. 4Final impression was made by open tray technique using addition silicone (Elite, Zhermack). It was poured with Type-IV dental stone (Ultrarock, Kalabhai Karson Pvt. Ltd.) and master cast was obtained. Abutments were surveyed milled and tried on patient mouth.( Fig 9) The mounted cast was then sent to laboratory for fabrication of cement retained milled porcelain fused to metal crown. 


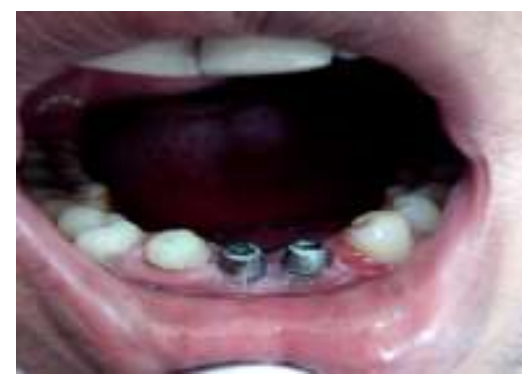

Fig-9

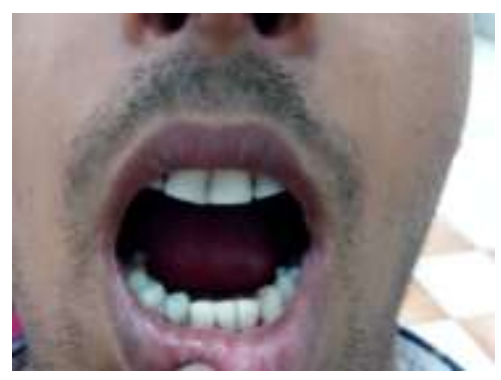

Fig-10

After 2 weeks of healing period, the implant was loaded with cement retained milled pfm crown [Figure 10]. Final cementation was performed with adhesive resin (RelyXUnicem transparent, 3M ESPE).

\section{Conclusion:-}

Immediate implant placement following tooth extraction has been found to be a feasible and predictable solution to tooth loss. This case report demonstrates minimally invasive surgical technique to Immediate implant placement in the esthetic zone achieve greater efficiency, ease of procedure and shorter time involved with minimum postextraction complications. This procedure is more difficult to execute than the conventional procedure. Therefore, we should be able to enhance the definitive treatment offered to the patient in regard to the treatment time, patient comfort, cost and esthetics.

\section{References:-}

1. Immediate implant placement and provisionalization with simultaneous guided bone regeneration in the esthetic zone. Chih-Long Chen, Chih-Ling Chang, Shih-Jung Lin. Journal of Dental Sciences (2011) 6, 53e60

2. Ebenezer V, Balakrishnan K, Asir RV, Sragunar B. Immediate placement of endosseous implants into the extraction sockets.J Pharm Bioallied Sci 2015;7 Suppl 1:S234-7.

3. Jay R.Beagle(2013)Indications and contraindications of immediate implant placement.Wiley Online Library.

4. JORDI Ortega-Martinez et al.(2012)Immediate implants followingtooth extraction:A Systematic Review.Med Oral Patol Oral Cir Bucal 1;17(2):e251-612)

5. Immediate Dental Implant Placement: Technique, Part 1.Authored by Gary Greenstein, DDS, MS, and John Cavallaro, Continuing Education, Course number 169. Dentalcetoday.com.

6. Chen ST, Darby IB, Reynolds EC. A prospective clinical study of non-submerged immediate implants: clinical outcomes and esthetic results. Clin Oral Implants Res.2007;18:552-562.

7. Sorni-Bröker M, Peñarrocha-Diago M, Peñar rocha-Diago M. Factors that influence the position of the periimplant soft tissues: a review. Med Oral Patol Oral Cir Bucal.2009;14:e475-e479.

8. Lekovic $\mathrm{V}$ et al. Preservation of alveolar bone in extraction sockets using bioabsorbable membranes. J Periodontol 1998; 69: 1044-1099.

9. Immediate implant placement in the esthetic zone - a clinical report. Shaily Tyagi,Divya Ratan Dahiya, Praful Mehra,Gaurav Issar. J Dent Specialities.2016;4(1):95-9 